\title{
L'ESSOR LITTÉRAIRE DE WILLIAM SHAKESPEARE EN FRANCE
}

\author{
Par \\ Rihame Mohammed Nabile Hifnawy* \\ R.hifnawy@mu.edu.eg
}

\section{Résumé}

Les liens étroits entre Shakespeare et la vie littéraire en France ne cessent pas de couler d'encre jusqu'à nos jours. Nombreux sont en fait les articles, les ouvrages et les études qui ont été consacrés à ces liens. Tantôt imité, tantôt traduit, Shakespeare a pu se frayer un chemin dans la conscience littéraire en France. Sans parler d'Alfred de Vigny ou Voltaire dont l'œuvre présente de fortes ressemblances avec le théâtre de William Shakespeare, des traductions, des articles de références, des représentations devant la Comédie Française ont créé ce qu'on pourrait appeler la fortune du grand dramaturge anglais en France. Nous tenterons donc de nous attaquer à cette fortune de Shakespeare en France à travers cette étude de réception littéraire. Nous nous attachons dans un premier temps de voir comment Shakespeare était au centre des préoccupations littéraires de l'époque. Il sera question dans un deuxième temps de nous attaquer aux adaptations et des traductions françaises de Shakespeare en France. Nous tentons enfin de voir de près comment l'œuvre théâtrale de Shakespeare a largement influencé le théâtre français au fils des siècles.

Mots-clés : Théâtre. Shakespeare. Fortune. Réception. Comédie française. Critique. Adaptations. Traductions. Ressemblances. Imitations.

Maître de Conférences, Département de français Faculté de Lettres, Université de Minia. 


\section{Introduction}

$L^{\prime}$ Ecole de Constance ${ }^{l}$ a accordé une importance particulière à la traduction en tant qu'un choix culturel qui nous informe sur les rapports étroits entre les différentes littératures et cultures. Autrement dit, choisir un écrivain ou une œuvre pour les traduire semble, pour certains comparatistes, être soumis à ce qu'on appelle «l'influence indirecte ${ }^{2} »$. Or, «le texte traduit est une sorte d'utopie, de non-lieu ${ }^{3} »$.

Quant à Shakespeare, jusqu'au XVIIIe siècle, il était quasiment absent du discours littéraire français. Le contexte politique, qui faisait de l'Angleterre et de la France deux pays voisins toujours en rivalité, retardait largement l'émergence de ce dramaturge anglais. De plus, le goût des dramaturges français du Grand siècle s'inspirait des Grecs et des Latins par respect du classicisme dominant tout au long de ce siècle. Il élabore une importance accordée à l'Antiquité, à l'Italie et à l'Espagne tout au long du grand siècle.

Il fallait attendre le siècle des Lumières pour que ce grand dramaturge anglais occupe une place à part en France. C'est avec Voltaire notamment qu'on ne discutait point de théâtre sans parler de l'auteur de Roméo et Juliette ${ }^{l}$. D'où vient le sujet de la présente étude où nous tentons de mettre l'accent sur cet état de

\footnotetext{
1 Roméo et Juliette est une tragédie de Shakespeare publiée en 1597. William Shakespeare, Roméo et Juliette, traduction de François-Victor Hugo, Euvres Complètes de Shakespeare, Pagnerre, Paris, 1868, pp.237-376.
} 
réception littéraire par les traductions et les imitations de Shakespeare en France.

Nous essayerons dans un premier temps de recenser aussi aisément que possible les traductions faites par les Français de l'auteur anglais. Puis nous nous attachons ici à voir comment les traductions et les adaptations ouvrirent la porte aux dramaturges français, à partir du XVIIIe siècle, pour s'inspirer, imiter, voire même de pasticher l'œuvre du grand dramaturge anglais.

Dans un deuxième temps, nous tenterons de fouiller historiquement les influences shakespeariennes sur le théâtre français. Nous évoquerons les noms des grands dramaturges français tels Voltaire, de Musset et bien d'autres encore qui se sont longuement inspiré de l'œuvre théâtrale de l'auteur d'Othello ${ }^{2}$.

Enfin, nous approfondirons notre analyse en étudiant la problématique des adversaires de Shakespeare en France. Tantôt pour des raisons politiques, tantôt pour des raisons de goût, certains français portent des jugements contre Shakespeare et son œuvre.

\section{Histoire de Shakespeare en France}

Les français et les anglais s'échangent l'amitié et la haine en même temps depuis longtemps. Ils sont "comme deux aimants

\footnotetext{
2 Othello ou Le Maure de Venise est une tragédie de Shakespeare, jouée pour la première fois en 1604. William Shakespeare, la tragédie d'Othello, traduction de François-Victor Hugo, Euvres Complètes de Shakespeare, Pagnerre, Paris, 1868, pp.237-397.
} 
prodigieux, ils s'attirent par côté et se fuient par l'autre, car ils sont à la fois ennemis et parents" ${ }^{\prime 4}$. Les relations étaient constantes et variées dans leurs pays, la France et l'Angleterre. En cette dernière, l'aspect littéraire suivait de très près ce qui se faisait en France. Les anglais se sont montrés fort curieux des moindres manifestations de la vie littéraire en France. Depuis longtemps, Les rois de France avaient des auxiliaires écossais qui parlaient l'anglais. Un grand nombre des étudiants anglais suivait des cours à l'université en France. Les auteurs anglais y venaient pour toucher de plus près les traditions classiques. Les souverains des deux pays s'envoyaient des ambassadeurs lettrés et poètes :

"Dès Charles VII de France, nous trouvons Alain Chartier envoyé en ambassade en Ecosse par le roi. L'humoriste Guagin représente en Angleterre le roi Charles VIII. De son côté, Henri VIII envoyait sir Francis Bryan et Sir Thomas Wyatt en France ${ }^{15}$

Sir Francis Bryan et Sir Thomas Wyatt étaient deux des plus élégants poètes du temps, versés dans la littérature antique, pénétrés de l'esprit de la Renaissance. Même le grand poète français de XVIème siècle, Ronsard, séjournait en Angleterre et se liait avec un des poètes les plus charmants de l'Angleterre, Sir David Lyndesay; il représentait à la cour d'Elizabeth la tragédie de Gorboduc. Grâce à sa civilisation remarquable, La France attirait les seigneurs anglais qui aimaient la littérature française. 
Mais jusqu'à présent sous le règne de Henri IV, on ignore encore Shakespeare ${ }^{6}$.

Quant à la littérature anglaise, elle est intimement liée au goût et à l'idéal littéraire français, tantôt acceptés, tantôt contestés, mais qui ne furent jamais ignorés en Europe. Pourtant la littérature dramatique atteint un développement complet en Angleterre près d'un siècle avant la France. Cet essor est dû en grande partie à Shakespeare dont la plupart des chefs-d'œuvre étaient produits à l'époque où naquit Corneille, un dramaturge français au XVIIe siècle. Shakespeare écrivait la plupart de ses œuvres durant l'époque d'Elisabeth quand l'Angleterre commençait à s'élever bien haut en splendeur et en prospérité. Ce règne doit être considéré comme le plus glorieux pour ce pays, de même que le fut cent ans plus tard en France le règne de Louis XIV. Shakespeare avait, de son vivant, acquis une certaine célébrité, mais très peu de gens imaginaient que ses œuvres seraient lues et représentées pendant des siècles encore.

Au dix-septième siècle, la France a commencé à découvrir Shakespeare, à partir de 1677 lorsque Saint-Evremond, très probablement, est le premier Français en parlant de lui dans l'Essai sur la comédie $^{7}$. En 1686, Adrien Baillet, à la fin de ses Jugements des savants sur les principaux ouvrages des auteurs, a fait allusion à lui lorsqu'il donnait une liste des plus notables Poètes des Isles Britanniques en langage vulgaire. En 1693, Shakespeare est célébré par Pierre-Antoine Le Motteux dans le 
Gentleman's Journal ${ }^{8}$. D'autre part, il faut citer également que Louis XIV, protecteur des lettres et des arts durant son époque, possédait une édition anglaise de son théâtre.

Au début du dix-huitième siècle, Shakespeare n'était connu jusque-là que d'un petit nombre de lettrés. S'ajoute à cela que, la traduction des œuvres de Shakespeare fut publiée en France. Le goût des Français lettrés pour l'anglais avait commencé à prendre une nouvelle allure. Puisqu'on s'intéresse à la culture anglaise dans son ensemble. À la faveur d'énormes entreprises de traductions, le théâtre de Shakespeare est redécouvert en France. C'est sur ces traductions remarquables, surtout celle de Letourneur, que s'appuie essentiellement les dramaturges français lorsqu'ils composent leurs adaptations de Shakespeare. Vers le milieu du dix-huitième siècle les adaptations des comédies de Shakespeare sont en vogue. En 1768, Barthe représente Les Fausse infidélités deuxième pièce imitée de Shakespeare a été jouée sur la scène de la Comédie-Française après La mort de César de Voltaire. De 1768 à 1804, pendant plus de vingt-cinq ans, les tragédies de Jean-François Ducis, adoptées de Shakespeare, ont été préférés sur la scène français.

Au début du dix-neuvième siècle, Madame de Staël a joué un rôle crucial dans l'ouverture de la France à Shakespeare, surtout avec la parution de son essai littéraire De l'Allemagne en 1814. Dans lequel, elle s'intéresse à la nécessaire ouverture du public français aux œuvres dramatique étrangère. En fait, Madame de 
Staël a puisé à l'ouvrage intitulé Le Cours de littérature dramatique $^{9}$ de Auguste Schlegel, paru en France en 1813. La seconde partie de cet ouvrage est consacrée aux théâtres modernes dont le plus éminents représentant est, selon Schlegel, Shakespeare. C'est également, Madame de Staël a rédigé son ouvrage De l'esprit de traductions en 1816, dans lequel, elle insiste sur la valeur culturelle de la traduction d'ouvrages anglais.

Il est évident que la redécouverte de l'œuvre Shakespearienne a coïncidé avec l'émergence d'une conscience romantique européenne. Son œuvre a contribué à donner naissance à une littérature dramatique actuelle en France. Notamment le théâtre de Shakespeare qui a apparu fidèle aux origines de l'art dramatique car toutes les couches de la société avaient leurs places sur la scène shakespearien.

Lorsque la France se libéralisait sous la Restauration, les romantiques s'émancipaient et commençaient à redécouvrir la littérature d'Outre-Manche. Stendhal, qui croit à la nécessité de l'existence d'un théâtre qui soit contemporain aux spectateurs, a contribué à une meilleure réception du théâtre de Shakespeare en France surtout avec la parution son ouvrage intitulé Racine et Shakespeare. Et Chateaubriand qui voit dans Shakespeare un mélange de défauts et de beautés, reconnait en lui un génie original. Pour lui, Shakespeare est "au nombre des cinq ou six écrivains qui ont suffi aux besoins et à l'aiment de la pensée; ces génies mères semblent avoir enfanté et allaité tous les autres ${ }^{10}$. 
On ne peut pas ignorer également que les dramaturges romantiques français ont inspiré à Shakespeare les sujets historiques dans les tragédies françaises modernes. Tous les dramaturges en quête de nouveauté ont commencé à s'intéresser au théâtre de Shakespeare, ce dieu de théâtre ${ }^{11}$. Et ses grandes œuvres étaient une source d'inspiration inexhaustible pour les écrivains qui y trouvent une ouverture sur de nouveaux horizons. C'est ainsi qu'en France, les œuvres de Shakespeare se sont rapidement frayées un chemin.

\section{Les traductions de Shakespeare en France}

Traduire le théâtre shakespearien en français, c'était transcrire, adopter et fonctionner ce théâtre dans les normes préexistantes du genre tragique français. Les réticences françaises ne continuent pas à négliger le succès de ce théâtre d'origine anglaise. Voltaire est le premier à jouer un rôle fondamental pour l'accession de Shakespeare en France. Pour lui, Shakespeare était loin d'être négligeable :

"C'est Shakespeare qui, tout barbare qu'il était, mit dans l'anglais cette force et cette énergie qu'on n'a jamais pu augmenter de puis sans l'outrer, et par conséquent sans l'affaiblir"'12.

Durant son séjour en Angleterre en 1725, Voltaire avait découvert le théâtre anglais et compris que Shakespeare avait "un génie plein de force et de fécondité, de naturel, de sublime, sans moindre connaissance des règles" ${ }^{13}$. Il assistait la représentation 
des plus célèbres tragédies et quelques drames historiques de ce grand dramaturge anglais à Londres. Dans ses Lettres anglaises ou Lettres Philosophiques, publiées en 1733, Voltaire lui rendait hommage. Et il est fort probable que ce grand philosophe français s'en inspire pour écrire Zä̈re, La Mort de César et Sémiramis où l'apparition d'un spectre renvoie à Hamlet qui eut sur lui une impression profonde, au point qu'il proposa de le traduire et d'en diffuser la version française dans la dix-huitième des Lettres anglaises ou Lettres philosophiques. C'était la première fois qu'on traduisait en français une pièce déjà célèbre, mais ignorée en France.

En 1731, Voltaire traduisait aussi et même imitait Julius Caesar de Shakespeare, ou La mort de César, mais d'une façon trop irrégulière. Il supprime les personnages féminins, les situations politiques et la conception de l'amour et de la liberté. Dans ses traductions, Voltaire essayait de remodeler le théâtre Shakespearien, jugé irrégulier voire barbare, pour correspondre au goût français. Mais jusqu'à présent, le public français n'avait à sa disposition que les rares fragments traduits par Voltaire. Et il n'existait aucune vraie traduction des auvres de Shakespeare.

Cependant, la fortune de Shakespeare en France est due en premier lieu à Pierre-Antoine de La Place. Ce dernier était le premier traducteur français à avoir publié en 1745 une traduction du théâtre de Shakespeare qui était beaucoup plus fidèle que celle de Voltaire. La Place essayait de mettre en lumière ou ressuscite 
la tragédie shakespearienne à un public de lecteurs et non pas à un public de spectateurs. Mais pour lui, le théâtre shakespearien est fondamentalement étranger, contraire aux règles d'Aristote. Et il juge les pièces de Shakespeare selon le goût de sa nation:

"Ce n'est donc point le goût de Shakespeare qu'il faut condamner, puisqu'il règne encore : c'est celui de la nation seule, qu'il faut censurer, s'il est mauvais, puisqu'elle a pu corriger ce goût et le rectifier, à notre exemple, depuis la mort de Shakespeare ${ }^{\prime 14}$.

Pourtant Grâce à sa traduction des œuvres théâtrales de Shakespeare, La Place contribue à instaurer la modernité dans le théâtre français, à renoncer à l'archaïsme et à permettre au public français de percevoir la richesse de l'imagination et les variantes des constructions. En outre, il réussit à convaincre le public d'entrevoir Shakespeare avec ménagement. Sa traduction est une œuvre utile qui popularisait Shakespeare et préparait le goût public de la France aux nouveautés dans le domaine théâtral. Désormais, l'intérêt pour la traduction de la littérature anglaise de Shakespeare commence ainsi à se développer en France.

Par ailleurs, parmi les traductions françaises de Shakespeare il y avait de bons ouvrages qui ont été bien reçus par le public. Nous pouvons évoquer la traduction de Pierre Letourneur (1737-1788) du théâtre shakespearien, en vingt volumes, de 1776 à 1782, sous le titre "Shakespeare traduit de l'anglais": c'est une traduction 
plus complète, supérieure aux précédentes et différente par son objectif qui comblait le texte traité par beaucoup de liberté.

Dans la préface de sa traduction, Letourneur entreprend "une traduction complète, la plus fidèle qu'il (lui) serait possible du théâtre entier de Shakespeare"15. Il propose une traduction au sens moderne, une traduction précise mais sans renoncer à la vocation poétique qui lui est inhérente. Il insiste, dans une tradition poétique, sur le fait de "conserver à l'original sa couleur, et au caractère sa vérité". ${ }^{16}$ En fait, sa traduction qui jouait un grand rôle dans la transformation de la perspective littéraire française garde fidèlement la liberté absolue qui distingue l'œuvre de Shakespeare. Par conséquent, la traduction des œuvres shakespeariennes, en dépit de ses lacunes et de la résistance du goût classique traditionnel, pénétrait dans la France.

C'est ainsi que Shakespeare devient au sein du discours critique français. Au début du dix-neuvième siècle, plusieurs traductions complètes lui sont attribuées.

En 1819, Bruguière de Sorsum, cousin par alliance d'Alfred de Vigny, qui s'intéressait avant tout à la littérature anglaise, traduisait des pièces de Shakespeare, publiées après sa mort en 1823. "il a déjà traduit six tragédies de Shakespeare d'une manière originale, littérale, et la seule qui révèle le génie

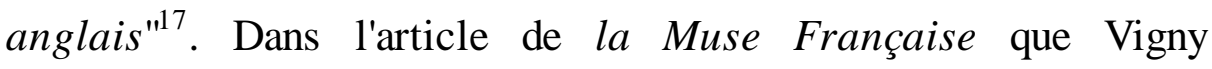
consacra à Bruguière, il parla de son talent comme traducteur de Shakespeare et son essai de rendre les nuances du style du 
dramaturge anglais sans faire violence à la langue française. Il y montra une admiration sincère pour son travail et sa méthode ${ }^{18}$.

François Guizot a publié, en 1821, chez Ladvocat la traduction des œuvres complètes de Shakespeare. Cette traduction était décisive, "elle devait mettre les lecteurs français en demeure de se prononcer sur Shakespeare tel qu'il est" ${ }^{19}$. Elle était précédée d'une étude biographique et littéraire sur Shakespeare, accompagnée de nombreuses notes de diverses pièces, et d'une tragédie et deux poèmes. A la suite de cette traduction, Shakespeare n'était plus méconnaissable en France, mais il est entré au sein de la littérature française grâce à sa brillante imagination, sa pensée forte et son génie. De plus, Benjamin Laroche a publié en 1839 une traduction des œuvres de Shakespeare, en deux volumes, chez Marchant qui est précédée d'une introduction sur le génie de Shakespeare par Alexandre Dumas. Cette traduction contenant trente-six drames est véritablement complète.

N'oublions pas de dire que cette entreprise demeure comme un modèle à suivre pour Vigny lui-même quand il traduisait des pièces de Shakespeare quelques années plus tard. De plus, une lettre d'A. De Champigneulle à Montlivault, datée du 29 novembre 1823, expliquait que Vigny s'était chargé des manuscrits de Bruguière et s'intéressait vivement à leur publication $^{20}$. Cette traduction lui a donné l'ambition de devenir à son tour le traducteur de Shakespeare et de donner à la France un 
Roméo et Juliette authentique. Alors, nombre de traducteurs et d'écrivains français ont traduit l'œuvre de Shakespeare au dixhuitième siècle. Grâce à leurs efforts, au seuil du dix-neuvième siècle la littérature de Shakespeare était devenue familière au public mais on ne peut pas ignorer également la traduction ponctuelle d'Alfred de Vigny et celle de Victor Hugo. Ceux qui ont essayé de présenter une traduction fidèle et intégrale de Shakespeare.

De ces diverses traductions, les plus appréciées du $\mathrm{XIX}^{\circ}$ siècle sont la traduction ponctuelle d'Alfred de Vigny et celle de Victor Hugo. D'abord, la traduction de Vigny peut être considérée comme une œuvre de traducteur ou plutôt d'adaptateur. Vigny commençait à lire Shakespeare dès son adolescence, peut-être plus tôt encore. Le témoignage le plus précieux sur les connaissances anglaises de Vigny était celui de Hervey qui, en 1844, a écrit dans son journal :

"Vigny parlait l'anglais correctement, mais avec un fort accent, et il était évident qu'il avait fait des études d'anglais longues et laborieuses. Quand je lui demandai où il l'avait appris, il répondit : de ma femme et de Shakespeare ${ }^{\prime 21}$.

Il est fort probable que l'œuvre de Shakespeare eut une influence décisive sur une partie de l'activité littéraire de Vigny. Vigny a lu l'œuvre de Shakespeare plus attentivement pour la traduire; il a pu apprendre à respecter les textes. C'est de 1826 à 1827 que Vigny commença à traduire Shakespeare. Sa traduction 
de Roméo et Juliette est une entreprise en collaboration avec Emile Deschamps qui traduisit les trois premiers actes et Vigny les deux derniers. Vigny, le traducteur, est resté fidèle à l'original mais avec une profonde différence dans le style. Il souhaitait produire une adaptation libre, mais Deschamps préférait une traduction littérale de cette pièce. La pièce n'a pas été publiée. Deschamps s'est décidé à retraduire les deux derniers actes et à publier toute l'œuvre. Quant à la traduction de Vigny, elle est restée, sans changement, pendant de longues années. En 1856, Vigne y essayait de la compléter mais il n'est jamais arrivé à la terminer. Après sa mort, le manuscrit passa à Louis Ratisbonne 22 puis à M. Tréfeu ${ }^{23}$ qui, en 1907, publie une partie dans la revue L'Italie et La France.

En 1839, Vigny publie, pour la première fois, la pièce du Marchand de Venise. Il l'avait achevée dès 1828 avec l'intention de la représenter sur scène. Mais ce projet a échoué aussi, et la pièce n'a jamais été représentée de son vivant. Il convient de noter que cette traduction n'était qu'une adaptation très réduite. Et en 1829, Vigny a terminé la troisième traduction de Shakespeare, Othello, dans laquelle, il ne s'agit plus d'une adaptation comme celle du Marchand de Venise, mais bien d'une traduction fidèle et complète. Il paraît complètement indépendant des adaptations françaises du drame. En général, Vigny a cherché à illustrer, dans sa traduction de Shakespeare, les théories classiques et romantiques. Quand on compare ses traductions de Shakespeare 
au texte anglais, on observe que les pièces de Shakespeare subissent une adaptation textuelle et structurelle.

L'autre meilleure traduction est celle de François-Victor Hugo vécu en exil avec son père. Il traduisait de 1858 à 1866 les œuvres complètes de Shakespeare. Victor Hugo a salué la traduction de son fils en disant : " le traducteur actuel sera, nous le croyons, le traducteur définitif ${ }^{124}$. Cette traduction était simple et fidèle où il a eu recours au vocabulaire riche, précis pour obéir au texte. Par souci de modernité, François-Victor Hugo traduisait les œuvres de Shakespeare en prose sans complaisance puriste pour la France ou puritaine pour l'Angleterre.

En effet, la réception de Shakespeare prend un nouveau tournant en France avec cette traduction. Particulièrement, François-Victor Hugo est le premier qui a fourni au public français la première traduction française intégrale des Sonnets de Shakespeare, toujours en accord avec l'inspiration romantique. Dans cette traduction il transmettait la richesse métaphorique de la langue de Shakespeare sans modifier les images qui paraissaient à l'époque choquantes. Il voulait produire une version qui se rapproche le plus possible de celle de l'auteur.

Il importe de rappeler ici qu'une nouvelle traduction d'Émile Montégut, vouée à une grande diffusion, a été publiée en 1867. Mais en dépit de sa connaissance plus approfondie de la langue anglaise, la traduction de Montégut se souciait peu du style et demeurait bien inférieure à celle de François Victor Hugo. A la 
fin de ce siècle, et grâce à toutes ces traductions, la réception de Shakespeare en France n'était plus complexe. Les traductions proposent au spectateur français la tragédie de Shakespeare, surtout Hamlet ${ }^{3}$. Ce qui témoigne de l'intérêt du XIXe siècle pour Shakespeare. Ces traductions ont fait connaitre Shakespeare à la France.

$\mathrm{Au}$ début du vingtième siècle, le nombre des traductions de Shakespeare est très nombreux. Pierre Messiaen, un homme de lettres français, est connu pour sa traduction de Shakespeare. Il a publié des œuvres complètes de Shakespeare de 1939 à 1943. Dans les années 1950, Yves Bonnefoy a consacré son activité de traducteur aux œuvres de Shakespeare. Pierre Leyris a proposé à Yves Bonnefoy de traduire Jules César ${ }^{4}$ et Hamlet, pour les Euvres complètes dont Leyris entreprenait l'édition. Le poète a accepté par désir de se mesurer à la langue anglaise et à sa poésie dont il percevait les grands moyens différents de ceux de la poésie française. Après la réussite de cette traduction, Bonnefoy est entré dans l'univers shakespearien avec une quinzaine d'ouvrages. En 1957, il a publié sa première traduction de Hamlet, pièce qu'il a retraduite à quatre reprises : en 1959, 1962,

\footnotetext{
${ }^{3}$ Hamlet est l'une des plus célèbres pièces de Shakespeare, la date exacte de sa composition n'est pas connu avec précision, la première représentation se situe sûrement entre 1598 et 1601. William Shakespeare, Hamlet, traduction de FrançoisVictor Hugo, Euvres Complètes de Shakespeare, Pagnerre, Paris, 1865, pp.101-199.

4 Jules César est une tragédie, probablement écrite en 1599 et publiée par la première fois en 1623. Elle est montée pour l'ouverture du Globe Théâtre à Londres. William Shakespeare, Jules César, traduction de Yves Bonnefoy, Euvres Complètes de Shakespeare, Le Club Français du Livre, paris, 1957.
} 
1978, 1988. Il a traduit de nombreuses pièces ainsi que des sonnets. Pour Bonnefoy, "traduire Shakespeare, une fois qu'on a commencé, devient rapidement un besoin, celui de comprendre l'œuvre, qui constitue un ensemble d'une cohérence certaine ${ }^{125}$. Dans toutes ses traductions, il a tenté de se rapprocher de la personne de l'auteur et de le déchiffrer à travers chacun des personnages de ses pièces. Ainsi il a pu recréer, par son propre mouvement, le texte de l'auteur en toute fidélité.

En effet, cette traduction riche est devenue remarquable grâce à la considérable production critique qui l'accompagne. Puisque Yves Bonnefoy a revécu l'expérience poétique de l'œuvre originale de Shakespeare et le pouvoir d'actualiser le texte original à travers la théorie herméneutique de l'explication et de l'interprétation du texte. En traduisant Shakespeare, il a essayé de dévoiler la structure profonde des auvres shakespeariennes dans le but d'en faire ressortir toute la modernité. Bonnefoy a pu effectivement atteindre son objectif principal par sa traduction de l'œuvre de Shakespeare : il a compris la totalité de cette œuvre et l'a mise en rapport avec sa propre œuvre poétique du XXème et du XXIème siècle.

Un autre interprète, apparu au vingtième siècle, a traduit aussi l'œuvre théâtrale de Shakespeare : c'est Jean-Michel Déprats. Il est maître de Conférences à l'université de Paris $\mathrm{X}$; il se consacre à la traduction des auteurs anglais et du théâtre élisabéthain. Il est devenu spécialiste de Shakespeare lorsqu'il a traduit près de trente 
pièces de cet auteur unique. Sa traduction de l'ensemble des pièces de Shakespeare a été publiée aux éditions de La Pléiade de 2000 à 2005 dans sept volumes, s'ajoute un album Shakespeare. Déprats a confirmé que l'œuvre shakespearienne était tout entière représentative :

"Écrit par un acteur pour des acteurs, c'est un texte où

l'ordre des mots, les rythmes, les images sont avant

tout porteurs de gestes, où les propriétés sensibles $d u$

verbe sont un instrument de jeu pour le comédien ${ }^{126}$.

Bref, la traduction de Déprats n'est qu'un processus de réécriture qui fait revivre l'original. Mais pour lui, la puissance de Shakespeare reste un mystère en soi, comme celle d'autres génies $^{27}$.

Il est évident que les traductions de l'œuvre shakespearienne, surtout celle de son théâtre, ont contribué à faire connaître au grand public ainsi qu'aux intellectuels français le grand poète anglais et ont offert aux lecteurs le contexte véritable de son œuvre. Dès lors, la nouvelle génération de dramaturges français était prête à opérer une grande évolution dans le théâtre français. Même le peuple a connu ainsi Shakespeare et son talent supérieur. En face des nouvelles générations, la stérile école classique a échoué misérablement, et sa chute n'en sert que davantage le triomphe des romantiques. D'où vient le sujet de la troisième partie de la présente recherche: l'influence de 
Shakespeare sur le développement du théâtre français et les tendances et la puissance des rénovateurs.

\section{Les influences shakespeariennes en France}

Dans les deux pays, le théâtre était la représentation vivante de l'esprit de la société. Il était le reflet de la civilisation morale : en Angleterre le théâtre représente les sentiments de liberté. Celui de Shakespeare ne connut ni entraves, ni bornes, il expose tout hardiment : la vie humaine et l'homme en général avec tous ses sentiments et toutes ses passions. Au contraire, en France, on n'avait pas cette énergie dans l'action, ni cette vivacité dans le sentiment, ni cette audace dans le langage. Le peuple y était soumis à la volonté souveraine d'un monarque absolu; la noblesse, seule considérée, était aussi soumise à l'étiquette la plus stricte; les idées nouvelles étaient vaincues et la littérature se reportait en arrière vers les auteurs anciens grecs et latins. Le théâtre classique reposait uniquement sur l'imitation des anciens. Il restait sous des règles imposées par Aristote, il subissait également l'influence de la société et de la cour de Louis XIV. Il ne pouvait pas être la manifestation du peuple mais il était une image de la cour. Dans le même ordre d'idée, on peut lire le théâtre de Corneille et celui de Racine. Ces deux grands auteurs, qui représentent dans leurs tragédies l'expression de la cour et de la haute société, ont étudié à fond le théâtre grec. De plus, l'activité de la plupart des dramaturges contemporains et des 
successeurs de ces deux auteurs n'était qu'une imitation des modèles établis par ces deux grands héros du classicisme français.

On doit convenir que le génie de Shakespeare se distingue de celui de ses contemporains français. la différence semble être énorme : son théâtre s'adapte à toutes les situations et à tous les personnages de la vie pour en reproduire toutes les splendeurs et toutes les misères. En plus, chacun de ses personnages se dirige d'après sa propre nature et se montre conformément aux circonstances où il se trouve, c'est que rien n'égale sa connaissance du cœur de l'homme, il trace des caractères et des sentiments. Tandis que Corneille et Racine produisent un théâtre classique de la cour royale et s'en tiennent constamment à certains personnages, toujours des rois ou des héros.

Quant à Molière, il s'inspirait également des Anciens : Grecs et Latins. Mais il faisait le portrait de l'homme de son temps. Il restait homme de Cour. A l'instar de Shakespeare, "Molière a connu de près la multitude et fréquenté la cour, subissant ainsi la double et salutaire influence du peuple avec lequel sa profession le mettait en contact, et de la société élégante. Tous deux ils étaient riches, ils aimaient le luxe, et n'affectaient nullement la gueuserie de la bohême littéraire" ${ }^{28}$. Ces grands auteurs étaient les représentants de la culture de leur pays et de leur époque. Ils étaient un rayonnement international et continuent d'être traduits et joués dans bien des pays. 
La réception théâtrale de Shakespeare se déroule grâce «aux tentatives de redéfinition des composantes esthétiques et dramatiques, qui affranchissent, à des degrés variables le théâtre des sujétions du goût classique et réorganisent la hiérarchie des valeurs littéraires ${ }^{29}$. Shakespeare a dégagé le théâtre français de la tragédie classique qui était une anomalie au milieu de la société moderne. Il a donné naissance à une nouvelle forme dramatique. Il a apporté la liberté, qui était longtemps exclue, au théâtre français. Shakespeare a créé en France un théâtre vraiment grand et national par son exemple.

$\mathrm{Au}$ dix-huitième siècle, de grandes œuvres, dignes d'admiration, furent publiées en France. Ces œuvres, qui ont contribué à ennoblir l'humanité, ont imité les modèles originaux des grands auteurs étrangers, comme Shakespeare. Durant ce siècle, il y avait des auteurs qui ont emprunté à Shakespeare ses sujets, et d'autres se sont inspirés de ses œuvres.

Voltaire était le premier de son époque à imiter souvent Shakespeare dans plusieurs de ses tragédies. Il observait les mœurs et les idées anglaises durant ses séjours en Angleterre. Il faut reconnaitre que l'Angleterre l'initiait aux grands principes de liberté qui étaient inconnus à cette époque en France. Il a apprécié l'esprit de liberté politique si chère au théâtre anglais, surtout à Shakespeare et son indépendance en matière d'art dramatique. Dans son Discours sur la tragédie de Brutus, Voltaire a avoué que le théâtre anglais est bien défectueux, mais dans ses pièces si 
monstrueuses, il y a des scènes admirables. Il a avoué également à son ami anglais, qu'«il a manqué jusqu'à présent à presque tous les auteurs tragiques de votre nation, cette pureté, cette conduite régulière, ces bienséances de l'action et du style, cette élégance, et toutes ces finesses de l'art qui ont établi la réputation du théâtre français depuis le grand Corneille $)^{30}$.

En Angleterre, Voltaire développait et enrichissait son génie littéraire. Il admirait les chefs-d'œuvre dramatiques de Shakespeare, puis il les a transmis dans son théâtre sans abandonner les règles classiques. Il sentait le profond contraste entre le théâtre anglais et le théâtre français; il a reconnu la richesse de vie, de liberté et d'action du drame shakespearien; il a perçu que c'était la nature, le cœur humain avec ses passions, la vie des nations et l'histoire qui donnaient l'inspiration aux poètes. Il a découvert dans la richesse de la vie et l'originalité du drame anglais de Shakespeare une source fertile propre à enrichir le théâtre français.

A Londres, Voltaire a composé sa tragédie Brutus qui est représentée le 11 décembre 1730, puis sa tragédie La Mort de César en 1733. Dans celles-ci qui ne sont qu'une imitation de Shakespeare, Voltaire a réalisé ce qu'il observe chez les anglais. C'est ce qu'il l'a avoué dans son discours sur la tragédie à cet égard :

"j'aurais du moins voulu transporter sur notre scène certaine beauté de la vôtre ${ }^{\prime 31}$. 
Voltaire s'est inspiré des idées anglaises pour créer une pièce abondante en idées républicaines. Il a voulu introduire les beautés admirables du théâtre shakespearien en France, mais la nature du peuple français n'était pas la même que celle du peuple anglais. Voltaire exposait ses idées et ses projets pour préparer le public à ces nouvelles tentatives.

Étant le génie du dix-huitième siècle, et le soutien du classicisme, voltaire a admiré Shakespeare qui lui a fourni l'idée du changement dans la forme de la tragédie :

«Il songe aux ombres que Shakespeare seul, dit-il, a su évoquer et faire parler avec succès. Il entrevoit la possibilité et les moyens d'agrandir l'action; enfin, il insiste pour qu'on emploie, à l'exemple de Shakespeare, un style adapté toujours à la situation $\gg^{32}$.

Dans La Mort de César de Voltaire, on découvre clairement l'influence de Shakespeare qu'il a imité dans de nombreux passages même en présentant les mêmes personnages. Pourtant Voltaire n'a pas renoncé à rester le fidèle disciple du système classique. Il a voulu réaliser le drame de Shakespeare mais les dispositions du pays l'ont obligé à traiter le sujet de ce drame bien différemment: il a avoué que le théâtre français n'est pas approprié à mettre en scène le champ de bataille et les différents combats ainsi que d'autres catastrophes. C'est parce que de tels 
événements ne sont ni des mœurs ni des règles de bienséance de la bonne société.

A l'instar des dramaturges classiques, Voltaire a refusé la représentation d'un assassinat sur la scène ou l'éclat d'une forte passion aux yeux du public, parce que ces actes sont des actes barbares et pleins de grossièreté. D'après Brutus et La Mort de César, voltaire a insisté à respecter les règles de bienséance du théâtre classique. Voltaire a voulu emprunter le grand mérite des pièces Shakespeariennes, il a essayé de corriger cet art pour paraître convenable à un public qui n'était pas habitué à ce merveilleux, mais il a échoué.

Nous constatons que l'influence de Shakespeare sur le théâtre de Voltaire était de plus en plus forte en 1732. Voltaire a représenté Éryphile, tragédie en cinq actes, imitation indirecte de Hamlet de Shakespeare dans certains détails. L'imitation manifeste de Shakespeare se remarque dans la scène où apparait l'ombre du roi mort, pareille à l'ombre du père de Hamlet. Toutes les deux viennent en effet révéler un crime commis et porter plainte contre une épouse infidèle et homicide ; toutes les deux réclament vengeance pour leur fils. C'est-à-dire, Shakespeare a fourni à Voltaire la matière de ses pièces. La similitude est grande entre les œuvres des deux poètes surtout dans les sujets et les détails. Dans le théâtre de Shakespeare, l'action se déploie largement, elle est pleine de vie ; l'inspiration s'y élève bien haut. Mais pour Voltaire, qui a donné toujours plus d'importance aux 
vers qu'au spectacle, «sa pièce se réduit à un simple et médiocre événement, elle est privée de mouvement, et des conversations bien faites, écrites en vers brillants, ne remplacent pas l'action, cette condition et cette vie du drame, or ici les personnages sont à peine animés $»^{33}$. Voltaire qui, a adopté d'abord dans Brutus la liberté de penser du drame shakespearien, a cherché, dans Éryphile, à s'assimiler encore davantage à Shakespeare.

En 1732, voltaire a écrit Zaïre qui était une imitation flagrante d'Othello de Shakespeare. Cette pièce, qui est considérée comme une adaptation libre d'Othello, évoque les mêmes thèmes: la jalousie, la tolérance et la relativité des religions. Voltaire a traité le thème religieux qui était l'un des thèmes préférés de l'auteur français. Alors que Shakespeare a évité toute discussion sur la religion dans sa pièce. Et ce qui constitue l'action dans la tragédie de Voltaire, c'est la confusion de Zaïre entre ses devoirs de fille chrétienne et sa tendresse pour Orosmane. Voltaire a introduit également l'élément chrétien avec le personnage de Lusignan. En effet, la tragédie shakespearienne a mis l'accent sur les personnages de Zä̈re et la foi. La tentative de voltaire d'imiter Shakespeare était plus considérable.

Dans ces écrits ainsi que dans quelques-unes de ses lettres, Voltaire a expliqué ses opinions et ses jugements sur les pièces dramatiques de Shakespeare. Il a avoué à M. Falkener, dans sa lettre antérieure à Zaïre, que «C'est au théâtre anglais que je dois la hardiesse que j'ai eue de mettre sur la scène les noms de nos 
rois et des anciennes familles du royaume. Il me paraît que cette nouveauté pourrait être la source d'un genre de tragédie qui nous est inconnu jusqu'ici et dont nous avons besoin ${ }^{34}$. Malgré tout cela, Voltaire n'a pas pu se libérer des idées anciennes. Pour ces raisons, nous devons avouer que Voltaire n'était pas l'homme qu'il fallait pour introduire Shakespeare en France, mais il était l'un des premiers qu'ont fait connaître le poète anglais en France ; il a préparé pour l'avenir cette influence qui devait un jour marquer le théâtre français.

En 1769, Jean François Ducis, un dramaturge français, s'est tourné vers l'imitation du drame de Shakespeare. Ducis ne comprenait pas la langue de Shakespeare, il a lu les pièces de ce dernier à partir des versions françaises de La Place et de Letourneur qui étaient assez infidèles. Mais Ducis a décidé de suivre l'esprit de Shakespeare, un esprit novateur, indépendant et hardi. Il a aussi composé la tragédie de Hamlet qui était une véritable nouveauté sur la scène française. En 1783, Ducis a donné une nouvelle imitation du Roi Lear ${ }^{5}$ dans laquelle il ne respectait à peu près rien de la pièce originale. Autrement dit, dans le théâtre de Ducis, toutes les pièces n'ont pas respecté l'inspiration véritable parce qu'il a réduit tous les héros de Shakespeare aux rôles qui confirment que «le théâtre doit avoir

\footnotetext{
${ }^{5}$ Le Roi Lear est une tragédie de Shakespeare probablement écrite entre 1603 et 1606 et jouée le 26 décembre 1606 en présence du roi Jaques $1^{\text {er }}$ d'Angleterre. William Shakespeare, Le Roi Lear, traduction de Pierre-Prime-Félicien Le Tourneur, Shakespeare traduit de l'anglois, tome 5, Mérigot Jeune, Paris, 1779.
} 
une certaine échelle de proportions qui grandisse les personnages, afin de les faire mieux saisir du public, ce qui n'empêche pas les peindre d'après nature ${ }^{35}$. Alors, l'idée de représenter les drames de Shakespeare sur la scène française par Ducis n'a pas abouti, parce qu'il n'a pas compris les ressources du drame anglais et il n'a pas réussi à représenter la puissante originalité de ce poète anglais. Rappelons que jusqu'alors, Shakespeare n'a été connu du public français que par des traductions infidèles ou par des adaptations comme celles de Ducis qui étaient plutôt des trahisons. Mais l'influence de Shakespeare sur l'art dramatique français se poursuivait et gagnait du terrain chaque jour.

$\mathrm{Au}$ dix-neuvième siècle, tout a sensiblement changé. Le rapprochement politique entre l'Angleterre et la France a largement dissipé l'atmosphère hostile qui régnait auparavant entre les deux pays, surtout après la publication de la nouvelle édition du Shakespeare de Letourneur crée par Guizot et de la Biographie universelle, une critique judicieuse de Shakespeare écrite par Villemain. Comme tous les partisans de Shakespeare, Guizot a cherché à déterminer son influence : il a abordé à ce propos la question des règles classiques. Il a admiré également en règles classiques le mélange du comique et du tragique et il a expliqué le génie de Shakespeare : le poète qui peut prendre ses acteurs dans tous les rangs de la société et les présenter dans toutes les situations ${ }^{36}$. C'était un épisode important où le 
dramaturge anglais a pu se frayer un chemin parmi les auteurs dramatiques français. L'effet des représentations anglaises de Shakespeare était très grand: son influence sur le théâtre français était inséparable de la réforme du théâtre, la jeune génération a lutté volontairement pour la réforme dramatique en invoquant Shakespeare comme un grand exemple.

La présence réelle de Shakespeare sur scène au XIX siècle était, au premier plan, grâce à Alfred de Vigny qui a créé en 1829 à la Comédie Française sa pièce le Maure de Venise, traduction fidèle et complète en vers d'Othello. Vigny, l'admirateur de Shakespeare, a refusé de rester étranger à cette vague d'enthousiasme qui a envahi le théâtre français. Il est parti pour Londres afin de confirmer sa présence aux représentations anglaises de Shakespeare qui lui ont donné une influence profonde et décisive ou comme l'affirme le discours suivant :

«Dans ce gigantesque Bazar, les arts du moins ne sont ni profanés ni oubliés. Shakespeare règne toujours; de Westminster à la Tour, son nom, son buste partout $\gg^{37}$.

C'était en octobre 1829 que le théâtre Français s'ouvrait pour la première fois à un héros shakespearien authentique, et non plus à de pâles imitations. Il semble que Vigny, figure influente du romantisme, ait choisi ${ }^{38}$ Othello pour imposer à la Comédie Française et aussi au public français une œuvre étrangère au 
théâtre classique: le Maure de Venise se rapprochait de la conception classique des unités de temps et de lieu, et l'action y est ramassée autour du caractère principal. Donc, c'était la pièce qui avait le plus de chance de réussir sur la scène de la Comédie Française et son succès faisait triompher les tendances nouvelles. Il a voulu convaincre le public français des mérites des conceptions théâtrales modernes en lui présentant une œuvre célèbre écrite en dehors du théâtre classique.

De 1826 à 1827, Vigny et son ami Emile Deschamps commençaient à traduire Roméo et Juliette où le premier a interprété seulement les deux derniers actes. Les deux amis présentaient leur adaptation à la Comédie Française, mais cette pièce n'est pas représentée. Plus tard, Deschamps se décidait à retraduire pour son compte les deux derniers actes et à publier le tout sous son nom. En 1856, Vigny s'est remis à sa traduction inachevée de Roméo et Juliette pour la refaire et la compléter, mais il n'est pas arrivé à terminer cette traduction jusqu'à sa mort. En 1828, Vigny achevait sa pièce Shylock, une adaptation en vers $d u$ Marchand de Venise, dans l'intention de la faire jouer mais aussi elle n'a jamais été représentée de son vivant. Ses traductions versifiées de Shakespeare s'inscrivent dans le drame romantique, de même que sa pièce Chatterton écrite en 1835. Elles restaient différentes des adaptations françaises du drame shakespearien. 
Jusqu'à la fin de sa vie Vigny resta fidèle à cet art Shakespearien. Sa pièce originale La Maréchale d'Ancre n'était qu'un drame romantique et shakespearien. L'influence du poète anglais sur cette pièce historique était profonde : Chez Vigny comme chez Shakespeare, la multiplicité des personnages appartiennent aux classes les plus diverses, on y voit la cour avec la haute et la petite noblesse, les parvenus et aussi les hommes du peuple. L'action est chargée à l'excès de détails et un grand nombre de scènes très différentes. Par ailleurs, Vigny s'inspirait de Shakespeare certaines règles théâtrales : les unités ont été fort maltraitées; pas d'unité de lieu, il y a autant de décors que d'actes. Vigny, toujours influencé par Shakespeare, a usé le mélange des genres; on y trouve le comique au milieu d'une scène tragique. La Maréchale d'Ancre demeure un drame précieux pour qui veut étudier l'évolution de Vigny comme un dramaturge qui se débarrassait de la tradition classique grâce à l'influence de Shakespeare. Enfin, on peut dire que c'est dans l'œuvre de Shakespeare que Vigny a puisé l'impulsion nécessaire pour écrire ses premières pièces de théâtre qui incarnent trois adaptations. Il y a trouvé «l'occasion de développer de grands thèmes repris dans le reste de son auvre, et d'assouplir son alexandrin ${ }^{39}$. Le besoin d'une révolution au théâtre a poussé la jeune génération des dramaturges du dix-neuvième siècle à se tourner vers le génie britannique qui a contribué à apporter les éléments d'un renouvellement à la scène française. Et à la fin de 
ce siècle, les spectateurs français commencent à s'habituer à voir ses pièces sur la scène.

A la faveur de nouvelles traductions précises et fidèles au texte original comme celles de Jean-Michel Déprats qui a traduit les Euvres complètes de Shakespeare pour la collection de la Pléiade, les dramaturges et les metteurs en scène français n'hésitaient pas à aborder les œuvres shakespeariennes sur la scène du vingtième siècle. Pour la première fois, au milieu de ce siècle Richard $I^{6}{ }^{6}$, une nouvelle pièce historique trouve sa place sur la scène grâce à Jean Vilar au premier Festival d'Avignon, parce qu'elle se concentre sur la politique, au-delà des questions de psychologie et de droit divin. Ce qui lui donne tout l'aspect d'un drame politique moderne. On ne peut pas oublier que Jean Vilar, le créateur du Festival d'Avignon qui est la plus importante manifestation du théâtre et du spectacle vivant du monde, s'occupait toujours à faire connaître au public français des classiques étrangers méconnus. Il a cité dans son livre $\mathrm{De} \mathrm{La}$ Tradition Théâtrale qu'il a pris l'exemple de Shakespeare parce que «Chacune de ses ouvres offre au régisseur trop imaginatif l'illusion et les tentations de la création ${ }^{40}$. En même temps que cette pièce permet de faire découvrir un grand classique étranger. La mise en scène de Richard II à Avignon donne à Vilar la possibilité d'avoir recours à de nouveaux moyens scéniques. La

\footnotetext{
${ }^{6}$ Richard II est une pièce historique de William Shakespeare, écrite en 1595 et publiée en 1597. William Shakespeare, Richard II, traduction de Jean Michel Déprats et Gisèle Venet, Euvres complètes de Shakespeare, tome2, Pléiade, Paris, 1995.
} 
nouvelle génération talentueuse n'a cessé d'être attirée par l'œuvre shakespearienne. Le théâtre élisabéthain n'est plus considéré comme inaccessible au public français. Les spectateurs s'habituent à voir ses pièces. Or, Shakespeare, le poète anglais, gagne du terrain en France.

\section{Conclusion}

En fait, Shakespeare dont la renommée a dépassé les frontières de l'Angleterre occupe une place à part dans la conscience littéraire française. Mais, si l'auteur du Marchand $d u$ Venise $^{7}$ était méconnu parmi les dramaturges français du Grand siècle à cause des règles du théâtre classiques qui étaient éloignées de son œuvre, son influence était sans égale parmi les écrivains du Siècle des Lumières. Grâce à Voltaire et à ses contemporains, Shakespeare a sensiblement influencé la vie littéraire en France au XVIIIe siècle.

Le XIXe siècle témoigne de la place qu'a occupée Shakespeare parmi les écrivains de ce siècle. On considère que les auteurs de la période romantique ont joué un rôle décisif dans la diffusion du théâtre shakespearien en France. L'œuvre de Shakespeare a été popularisée en France par la publication et la représentation d'imitations et de traductions. Bref, l'influence de Shakespeare sur le théâtre français devient profondément profitable et les dramaturges français ont essayé d'imiter le théâtre

\footnotetext{
${ }^{7}$ Marchand du Venise est une pièce du théâtre de Shakespeare écrite entre 1596 et 1597. William Shakespeare, Le Marchand de Venise, traduction de M.Guizot, EEvvre complète de Shakespeare, tome VI, Didier et Cie, Paris, 1862.
} 


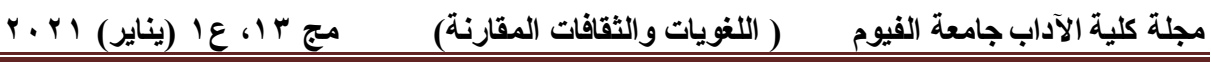

shakespearien. Et Shakespeare prend ainsi l'essor littéralement en France. Ce qui lui permet aussi de jouir d'une grande célébrité partout. 


\section{Les références :}

1 Les études littéraires au vingtième siècle s'intéressent aux théories de réception, qui sont centrées sur le lecteur et son rôle dans la réception des œuvres littéraires. À partir des années soixante-dix le terme «réception» ${ }^{1}$ est de plus en plus employé, dans toutes les langues; il a remplacé le mot indiquant les des études comparatistes à leur début, «influence». Et les théories de la réception ont mis en évidence le rôle essentiel du lecteur dans la production littéraire, puisque ce lecteur est bien présent en arrière plan des théories de la réception, avec sa subjectivité et la singularité de sa manière de lire. Le lecteur réel est au cour de toute expérience vivante et sensible des œuvres, où l'écrivain évoque l'incidence de ses expériences intimes de lecture dans son activité d'écriture. Dès lors, le lecteur participe à l'actualisation du sens de l'œuvre et s'emploie à déclencher le processus de la réception. Hans-Robert Jauss, théoricien de la littérature, est considéré comme l'un des principaux représentants des théories de la réception, surtout «l'esthétique de la réception» qui est fondée sur le lecteur. Cette théorie présente un intérêt majeur pour reconstituer l'horizon d'attente du premier lecteur puis à comparer les situations historiques des lecteurs successifs en mettant en relation les attentes et les opinions du lecteur, et les normes esthétiques et sociales. Certainement, la théorie de la réception est basée sur l'impression du lecteur qui reproduit les œuvres littéraires selon ses valeurs culturelles et sociales. Voir à cet égard : JAUSS Hans Robert, pour une esthétique de la réception, Paris Gallimard, 1990, p.63.

2 Pierre Brunel, Claude Pichoix, A.-M. Rousseau, Qu'est-ce que la littérature comparée, Armand Colin, Paris, 1983, p.51.

${ }^{3}$ Daniel-Henri Pageaux, Littérature générale et comparée, Armand Colin, Coll. «Cursus », 1994, p. 41.

${ }^{4}$ Edme Jaques Benoit Rathery, Des relations sociales et intellectuelles entre la France et l'Angleterre, depuis la Conquête des Normands jusqu'à la Révolution Française , Paris, Dubuisson et Cie, 1856, p.3.

5 Johnson Alfred Forbes, Études sur la littérature comparée de la France et de l'Angleterre à la fin du XVIIème siècleLafosse, Otway Saint-Real, origines et transformation d'un thème tragique, , Genève, Slatkine, 1971, p.248.

${ }^{6}$ Ibid. 
${ }^{7}$ Dubeux Albert, les traductions françaises de Shakespeare in Etudes françaises, Paris, LES BELLES LETTRES, 1928, p.3.

8 Voir à cet égard : Lucette Desvignes-Parent, Marivaux et l'Angleterre: essai sur une création dramatique originale, Paris, C.Klincksieck, 1970, p.137.

9 «Le cours de littérature dramatique » de August Wilhelm Schlegel est la reproduction des leçons publiques données à Vienne, en 1808, par l'illustre critique allemand et publiées de 1809 à 1811. Ce cours eut un retentissement énorme, non seulement en Allemagne mais en France. Mme de Staël y assistait; elle avait pour Wilhelm Schelgel une grand admiration, elle l'avait attiré à Coppet dès 1805, en lui confiant l'éduction littéraire de ses enfants, et bien des idées, sans doute, sur la philosophie de l'art, avaient été élaborées en commun par ces deux esprits d'élite. Auguste Wilhelm Schlegel, Cours de littérature dramatique, traduit par Mme Necker de Saussure , Tome 1, Paris, Librairie Internationale, 1865, p.V.

${ }^{10}$ François -René Chateaubrand, Essai sur la littérature anglaise, Paris, Eugène et Victor Perraud Frères éditeurs, 1849, p.122.

${ }^{11}$ Victor Hugo l'a mentionné dans la préface de son livre Cromwell Hernani. Voir à cet égard : Victor Hugo, Cromwell Hernani, Paris, Librairie Ollendorff, 1912, p.17.

${ }^{12}$ Voltaire, Discours de M.de Voltaire à sa réception à l'Académie

Française, œuvres complète de Voltaire, Paris, Furne, 1935, p.3.

${ }^{13}$ Voltaire, Lettres philosophiques ou lettres anglaises, Paris, Garnier, 1956, p.104.

${ }^{14}$ Pierre-Antoine De La Place, Le Théâtre Anglais, T1, Royaume-Uni, Londres, 1747, p. XV.

15 Pierre LETOURNEUR, Préface de Shakespeare traduit de l'anglais, Genève, Jacques Guy, 1990, p.14.

${ }^{16}$ Paul Van Tieghem, La Découverte de Shakespeare sur le continent, Paris, Sflet, 1947, p.211.

${ }^{17}$ Alfred de Vigny, Correspondance (1822-1849), TI, éd. Léon séché, Paris, Renaissance du livre, 1910, p.11.

18 Voir à cet égard: Alfred de Vigny, Euvres posthumes de M. le baron de Sorsum, in La Muse Française $\mathrm{n}^{\circ}$, Paris, Huward-Coucier, Janvier 1824, p.63.

${ }^{19}$ François Guizot, Euvre complète de Shakespeare, Paris, Didier et Cie, 1864, p.3. 
${ }^{20}$ A. Cherel, Autour de Bruguière de Sorsum, in revue de Littérature Comparée VII, Paris, E. Champion, (janvier .-mars 1927), p.163.

${ }^{21}$ Léon Seche, Alfred de Vigny, TII, Paris, Mercure de France, 1913, p.62.

${ }^{22}$ Louis Gustave Fortuné Ratisbonne (1827-1900) est un homme de Lettres français. Son travail le plus important est une traduction en vers de La Divine Comédie. Il est l'exécuteur testamentaire littéraire d'Alfred de Vigny, dont il publie Les Destinées (1864) et le Journal d'un poète (1867).

23 Etienne Victor Tréfeu (1821-1903) est un librettiste Français. Il est connu principalement pour sa fonction d'administrateur du théâtre de la Gaité à partir de 1873 .

24 Victor Hugo, Euvre complète de Shakespeare, traduction par François-Victor Hugo, préface de Victor Hugo, Paris, Pagnerre, 1865, p.30.

${ }^{25}$ Yves Bonnefoy, Traduire Shakespeare, in La Communauté des traducteurs, Strasbourg, Presses Universitaires de Strasbourg, 2000, p.86.

${ }^{26}$ Jean-Michel Déprats, La troisième langue, in Théâtre aujourd'hui, $\mathrm{n}^{\circ} 6$, Shakespeare, la scène et ses miroirs: Hamlet, la Nuit des rois, Paris, CNDP, 1998, p.50.

${ }^{27}$ Jean-Michel Déprats, Entretien réalisé par Jean-Pierre Léonardini, in L'Humanité, 19 Août 2002. Consulté le 4 octobre 2014 sur Site internet http://www.humanite. fr/node/270055

${ }^{28}$ Paul Starfer, Molière et Shakespeare, Paris, Libraire Hachette et Cie, 1887, pp.38-39.

${ }^{29}$ Christine Roger, "présentation », in Revue germanique internationale, $\mathrm{n}^{\circ} 5$, Parsi, CNRS, 2007, p.9; consulté le 4 mai 2014 sur site internet https://journals.openedition.org/rgi/163

${ }^{30}$ Voltaire, Discours sur la tragédie de Brutus, in ceuvres complètes de Voltaire, TI, Paris, Firman Didot frères, fils et CIE, 1843, p.149.

${ }^{31}$ Voltaire, Discours sur la tragédie de Brutus, op.cit, p.149.

${ }^{32}$ Albert Carroir, Histoire de l'influence de Shakespeare sur le théâtre français, Bruxelles, imprimerie de th. Lesigne, 1856, p.41.

${ }^{33}$ Albert Carroir, Histoire de l'influence de Shakespeare sur le théâtre français, op.cit, p.49.

${ }_{34}$ Ouvrages complète de Voltaire , $\mathrm{t} 2, \mathrm{p} .346$.

35 Albert Carroir, op.cit., p.169. 
${ }^{36}$ Voir à cet égard François Guizot, Shakespeare et son temps, Paris, Didier, 1852. Et François Guizot, Euvres complètes de Shakespeare, op.cit., p.5

${ }^{37}$ Alfred de Vigny, Lettres inédites de Alfred de Vigny au marquis et à la marquise de La Grange (1827-1861), publ. Avec une intr. Et des notes par Albert de Luppé, Paris, L.Conard, 1914, p.64.

${ }^{38}$ Il est important de citer ici que Vigny a expliqué pourquoi il a choisi Othello dans «La lettre à Lord sur soirée du 24 Octobre 1829 (date de la création (et sur un système dramatique » qui sert de préface à l'édition de la pièce.

${ }^{39}$ Fernarde Bassan avec la Collaboration de Sylvie Chevalley, Alfred de Vigny et la Comédie Française, Paris, Editions Jean Michel Place, 1984, p.14.

40 Jean Vilar, De La Tradition Théâtrale, Paris, L'arches, 1955, p.63. 


\section{Bibliographie}

\section{I- Ouvrages consacrés sur Shakespeare}

1- François - René Chateaubriand, Essai sur la littérature anglaise, Eugène et Victor Perraud Frères éditeurs, Paris, 1849.

2- Guizot (François), Euvre complète de Shakespeare, Didier et Cie, Paris, 1864.

3- Guizot (François), Shakespeare et son temps, Didier, Paris, 1852.

4- Hugo (Victor), Euvre complète de Shakespeare, traduction par François-Victor, préface de Victor Hugo, Pagnerre, Paris, 1865.

5- LETOURNEUR (Pierre-Prime-Félicien), Préface du Shakespeare traduit de l'anglais, édition critique par Jacques Guy, Droz, Genève, 1990.

6- Starfer (Paul), Molière et Shakespeare, Libraire Hachette et Cie, Paris, 1887.

7- Van Tieghem (Paul), La Découverte de Shakespeare sur le continent, Sflet, Paris, 1947.

II- Articles portant sur Wiliem Shakespeare

1- Albert (Dubeux), les traductions françaises de Shakespeare in Etudes françaises, LES BELLES LETTRES, Paris, 1928.

2- Bonnefoy (Yves), Traduire Shakespeare, in La Communauté des traducteurs, Presses Universitaires de Strasbourg, Strasbourg, 2000. 


\section{III- Ouvrages critiques sur la littérature comparée}

1- Alfred Forbes (Johnson), Études sur la littérature comparée de la France et de l'Angleterre à la fin du XVIIème siècle, Lafosse, Otway Saint-Real, origines et transformation d'un thème tragique, Slatkine, Genève, 1971.

2- Brune (Pierre), Pichoix (Claude), Rousseau (A.-M)., Qu'estce que la littérature comparée, Armand Colin, Paris, 1983.

3- Carroir (Albert), Histoire de l'influence de Shakespeare sur le théâtre français, imprimerie de th. Lesigne, Bruxelles, 1856.

4- De La Place (Pierre-Antoine), Le théâtre Anglais, TI, Royaume-Uni, Londres, 1747.

5- JAUSS (Hans Robert), Pour une esthétique de la réception, Gallimard, Paris, 1990.

6- Pageaux (Daniel-Henri), Littérature générale et comparée, Armand Colin, Coll. «Cursus », Paris, 1994.

\section{IV-Ouvrages généraux}

1- BASSAN (Fernande) avec la Collaboration de Chevalley (Sylvie), Alfred de Vigny et la Comédie Française, Editions Jean Michel Place, Paris 1984.

2- Cherel (A.), Autour de Bruguière de Sorsum, in revue de Littérature Comparée VII, E.Champion,Paris, (janvier .mars 1927).

3- Desvignes-Parent (Lucette), Marivaux et l'Angleterre: essai sur une création dramatique originale, C.Klincksieck, Paris,1970. 
4- Hugo (Victor), Cromwell Hernani, Librairie Ollendorff, Paris, 1912.

5- Rathery (Edme Jaques Benoit), Des relations sociales et intellectuelles entre la France et l'Angleterre, depuis la Conquête des Normands jusqu'à la Révolution Française, Dubussion et Cie, Paris, 1856.

6- Schlegel (Auguste Wilhelm), Cours de littérature dramatique, traduit par Mme Necker de Saussure , Tome 1, Librairie Internationale, Paris, 1865.

7- Séché (Léon), Alfred de Vigny, TII, Mercure de France, Paris 1913.

8- Voltaire (François-Marie Arouet), Lettres philosophiques ou lettres anglaises, Garnier, Paris, 1956.

8- Voltaire (François-Marie Arouet), Discours de M.de Voltaire à sa réception à l'Académie Française, œuvres complètes de Voltaire, Furne, Paris, 1935.

9- Vrinat-Nikolov (Marie), Miroir de l'altérite, la traduction, Ellug, Grenoble, 2006.

10- Vigny (Alfred de), Correspondance (1822-1849), TI, éd. Léon séché, Renaissance du livre Paris, , 1910.

11-Vigny (Alfred de), Guvres posthumes de M. le baron de Sorsum, in La Muse Française ${ }^{\circ}$ 7, Huward-Coucier, Paris, Janvier 1824.

12-Vigny (Alfred de), Lettres inédites de Alfred de Vigny au marquis et à la marquise de La Grange (1827-1861), L.Conard, Paris, 1914. 
13-Vilar (Jean), De La Tradition Théâtrale, L'arches, Paris, 1955.

\section{$\underline{\text { V-Articles généraux }}$}

1- Auger (L.S.), Avertissement sur Ducis, in euvres Jean François Ducis, t1, Ledentu, Paris, 1839.

2- Déprats (Jean-Michel), La troisième langue, in Théâtre aujourd'hui, $\mathrm{n}^{\circ} 6$, Shakespeare, la scène et ses miroirs : Hamlet, la Nuit des rois, CNDP, Paris, 1998.

3- Déprats (Jean-Michel), Entretien réalisé par Jean-Pierre Léonardini, in L'Humanité, 19 Août 2002. Site internet http://www.humanite.fr/node/270055

4- Roger (Christine), "présentation", in Revue germanique internationale, $\mathrm{n}^{\circ}$ 5, CNRS, Paris, 2007 ; site internet https://journals.openedition.org/rgi/163

5- Voltaire (François-Marie Arouet), Discours sur la tragédie de Brutus, in ouvres complètes de Voltaire, TI, Paris, Firman Didot frères, fils et CIE, Paris, 1843. 


\section{ملخص}

حتى يومنا هذا لازال يكتب الكثير عن الروابط الوثيقة بين شكسبير والحياة الأدبية في فرنسا. وهناك في الواقع العديد من المقالات والكتب والدراسات

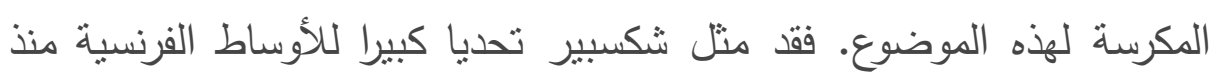
أواخر القرن السابع عشر وبداية القرن الثامن عشر، فبعد الترجمات المتلاحقة لأعماله المسرحية شرع النقد الفرنسي في تناول مسرحه الأمريكي الذي جعل الكاتب الانجليزي ذائع الصيت في الوسط الأدبي والنقدي الفرنسي ودون الحديث عن الفريد دي فيني او فولتير اللذان حاكي مسرحها شكسبير، تعددت مصادر رصيد شكسبير في فرنسا من عروض لمسرحياته امام الكوميديا الفرنسية ذاتها وكذلك الترجمات والرصد الأدبي لمؤلفه ومتابعاته النقدية في الصحف والمجلات. إذا تتصدي هذه الدراسة لرصيد المسرحي لثكسبير في فرنسا وتتخذ ثلاثة أبعاد. سنحاول في الأول منها توضيح كيف دخلت أعمال شكسبير المسرحية إلى فرنسا وكيف نم تلقيها. ثم نحاول بعد ذلك نوضيح أهم الترجمات

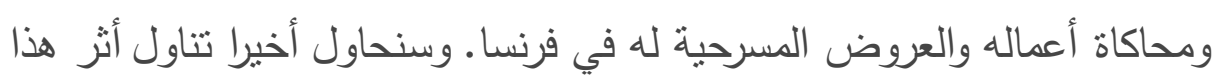
الكاتب الانجليزي على كتاب المسرح الفرنسي على مر العصور 